\title{
REFLEXÕES SOBRE A DISCIPLINA DE HISTÓRIA DA PSICOLOGIA NO ESTADO DO MATO GROSSO DO SUL
}

\author{
Fernanda Mongelli Honda Flores ${ }^{1}$; http://orcid.org/0000-0002-0545-8585 \\ Bianca Stephani Rodrigues²; http://orcid.org/0000-0002-4495-9390 \\ Alline Cavalheiro Sales ${ }^{3}$; http://orcid.org/0000-0003-1435-2181 \\ Flávio Henrique Nantes Edges ${ }^{4}$; http://orcid.org/0000-0002-8184-9983 \\ Rodrigo Lopes Miranda ${ }^{5}$; http://orcid.org/0000-0003-3222-7368
}

Paulo Coelho Castelo Branco ${ }^{6}$; http://orcid.org/0000-0003-4071-3411

\begin{abstract}
Resumo
Este artigo objetivou analisar a organização do ensino da disciplina de História da Psicologia, baseando-nos em cursos de graduação em Psicologia, no estado do Mato Grosso do Sul. Inicialmente, inventariaram-se as instituições que possuem cursos de Psicologia, acessando os seus sites e aplicando questionários aos seus coordenadores. Em seguida, organizaram-se algumas características gerais dos cursos e das instituições anuentes. Posteriormente, discutiu-se como o ensino da História da Psicologia auxilia na formação de estudantes, introduzindo-os ao estudo da Psicologia e às suas contendas científicas e profissionais. Ele contribui, com críticas, ao que se empreende no presente, embora apresente dificuldades de proporcionar algo introdutório mesclado a reflexões avançadas em uma disciplina. Por fim, observou-se que ele pode ser alocado e articulado com outras disciplinas, fazendo interface com os domínios da Ontologia, Epistemologia, Lógica e Ética. Palavras-chave: Ensino Superior; Disciplina; História da Psicologia; Formação do Psicólogo; Universidades.
\end{abstract}

\section{Reflections on History of Psychology's course in Mato Grosso do Sul}

\begin{abstract}
This article aimed to analyze the History of Psychology subject teaching organization, based on the Psychology undergraduate courses in Mato Grosso do Sul. Initially, institutions with Psychology courses were inventoried; their websites were accessed and surveys were applied to their coordinators. Ten, some general characteristics of these courses and institutions were organized. Afterwards, it was discussed how the teaching of History of Psychology helps the students training, introducing them to Psychology and its scientific and professional strife. It also contributes with criticism on what have been done in the present, although it has some boundaries providing introductory issues mixed to advanced reflections on Psychology. Finally, it was observed that this teaching could be placed and articulated with other subjects, interfacing with Ontology, Epistemology, Logics and Ethics domains.
\end{abstract}

Keywords: Higher Education; Discipline; History of Psychology; Psychology Training.

1 Universidade Católica Dom Bosco - UCDB - Campo Grande - MS - Brasil; mhflores.fer@gmail.com

2 Universidade Católica Dom Bosco - UCDB - Campo Grande - MS - Brasil; biancarodrigues123@hotmail.com

3 Universidade Católica Dom Bosco - UCDB - Campo Grande - MS - Brasil; cavalheiro.alline@gmail.com

4 Universidade Católica Dom Bosco - UCDB - Campo Grande - MS - Brasil; flavioetges@gmail.com

5 Universidade Católica Dom Bosco - UCDB - Campo Grande - MS - Brasil; rlmiranda@ucdb.br

6 Universidade Federal da Bahia - UFBA - Bahia - BA - Brasil; pauloccbranco@gmail.com 


\section{Reflexiones acerca de la asignatura de Historia de la Psicología en Mato Grosso do Sul}

\section{Resumen}

Este artículo tuvo como objetivo analizar la organización de la enseñanza de la asignatura de Historia de la Psicología, basándose en cursos de grado en Psicología, en el estado de Mato Grosso do Sul. Al principio, se inventariaron las instituciones que poseen cursos de Psicología, accediendo a sus sitios web y aplicando cuestionarios a sus coordinadores. A continuación, se organizaron algunas características generales de los cursos y de las instituciones anuentes. Después, se discutió cómo la enseñanza de la Historia de la Psicología auxilia en la formación de estudiantes, introduciéndolos al estudio de la Psicología y a sus contiendas profesionales y científicas; contribuye, con críticas, a lo que se emprende en el presente; presenta dificultades de proporcionar algo introductorio y múltiple, mezclado con reflexiones avanzadas en una disciplina; puede ser asignado y articulado con otras disciplinas; puede hacer interfaz con los dominios de la Ontología, Epistemología, Lógica y Ética.

Palabras clave: Enseñanza Superior; Asignatura; Historia de la Psicología; Formación del Psicólogo; Universidades.

\section{Introdução}

A Psicologia se firmou, como uma ciência, a partir de uma congregação de várias disciplinas que versavam sobre questões relacionadas ao psiquismo e aos métodos para acessá-lo, pesquisá-lo, compreendê-lo em suas manifestações e promover intervenções, nos mais diversos âmbitos relacionais e institucionais (Gundlach, 2012). Com efeito, no Brasil, a Psicologia é vista como um campo científico e profissional que enseja uma organização formativa institucional de psicólogos, em Instituições de Ensino Superior (IES) (Mancebo, Vale \& Martins, 2015). Nesse sentido, do conjunto de disciplinas que compõem esse quadro formativo, destacamos a História da Psicologia como um componente importante à graduação do psicólogo.

Amparado pelo estudo de Gundlach (2012), entende-se o termo disciplina (da História da Psicologia) como um quadro organizado em uma estrutura administrativa de ensino superior, programado para perpassar e ensinar conteúdos relacionados a um conhecimento, a partir de leituras, reflexões e discussões que afetam os planos de ações dos membros de uma classe profissional. Além disso, uma disciplina pode ser compreendida como a formação de discípulos e docentes para pensar, atuar e desenvolver um escopo de conhecimentos teóricos e práticos, em uma comunidade científica - de historiadores da Psicologia, neste caso.

Recentemente percebe-se, pois, um interesse pelo ensino de História da Psicologia, como parte do esforço de compreender mecanismos de disciplinarização do campo, no Brasil e alhures (Vaugh-Blount, Rutherford, Baker \& Johnson, 2009, Stevison, Biggs,
\& Abramson, 2010; Fierro, 2014; Krampen, 2015; Merced, Stutman \& Mann, 2017). Neste cenário, a disciplina de História da Psicologia adquire importância para a formação em Psicologia, incidindo sobre uma formação crítica, influindo como motivadora do desenvolvimento científico e profissional. Entretanto, estudos internacionais - sobretudo aqueles que se referem ao Canadá e aos EUA - indicam que as disciplinas de História da Psicologia não são, necessariamente, essenciais para que o estudante obtenha o título de psicólogo ${ }^{7}$ (Barnes \& Greer, 2014).

Diferentemente do Canadá e dos EUA, o ensino de História da Psicologia, nos cursos brasileiros de graduação, atualmente, é obrigatório. O Ministério da Educação e Cultura instituiu, em 2011, as novas Diretrizes Curriculares Nacionais (DCNs) para o curso de Psicologia (Naves, Silva, Peretta, Nasciutti, \& Silva, 2017). Em seu $5^{\circ}$ artigo, elas indicam, explicitamente, que o ensino de fundamentos epistemológicos e históricos é imperativo na formação do futuro psicólogo, como forma de avaliar, criticamente, as linhas de pensamento, em Psicologia (Ministério da Educação e Cultura, 2011).

A legislação atual sobre a formação em cursos nacionais de Psicologia aponta para a institucionalização da história, nos departamentos e cursos de Psicologia. Entretanto, com base nesse recente cenário e nos apontamentos de Loureiro e Baptista (2007),

7 Os autores citados, em suma, apontam que a disciplina de História da Psicologia não é necessária para a obtenção de título, tanto na graduação (undergraduate) quanto na pós-graduação (graduate). Como o sistema de ensino canadense e estadunidense se diferencia do brasileiro, particularmente no fato da graduação não necessariamente ter função profissionalizante, optou-se por dizer "título de psicólogo", para facilitar o caráter comunicativo. 
nota-se uma carência de estudos que auxiliem a mapear as características do ensino de História da Psicologia, no país. Diante desse panorama, esta pesquisa objetivou analisar o ensino da disciplina de História da Psicologia, em cursos de graduação em Psicologia, nas IES do estado do Mato Grosso do Sul (MS).

\section{Método}

O estudo se orienta como um tipo de pesquisa exploratório-descritiva, dada a inexistência de pesquisas desse âmbito, em MS. Recorreram-se a estratégias mistas de pesquisa, qualitativas e quantitativas, tendo em vista a natureza dos dados produzidos.

\section{Participantes}

Participaram do estudo, com consentimento livre e esclarecido, cinco coordenadores de cursos de graduação em Psicologia de IES de MS.

\section{Materiais}

Foram utilizados dois questionários de preenchimento online, produzido pelos pesquisadores. Um questionário era destinado aos coordenadores de curso de graduação em Psicologia e outro orientado aos professores de disciplinas de História da Psicologia.

\section{Procedimentos}

Inicialmente, levantaram-se as IES que possuíam cursos de graduação em Psicologia, no estado. Foi identificada a existência de dez cursos, a saber: Universidade Católica Dom Bosco (UCDB), Universidade para o Desenvolvimento da Região do Pantanal (UNIDERP), Centro Universitário da Grande Dourados (UNIGRAN), Universidade Federal de Mato Grosso do Sul - Campus Campo Grande (UFMS), Universidade Federal de Mato Grosso do Sul - Campus Pantanal (UFMS - CPAN), Faculdade Anhanguera de Dourados (FAD), Universidade Federal de Grande Dourados (UFGD), Centro Universitário da Grande Dourados - Capital (UNIGRAN), Universidade Federal de Mato Grosso do Sul - Campus Paranaíba (UFMS - CPAR) e Faculdades Integradas de Três Lagoas (AEMS).

Em seguida, foram pré-selecionados os coordenadores de cada curso e os professores que ministravam a disciplina de História da Psicologia (ou uma equivalente). Por meio de e-mails, encaminhou-se uma carta convite para a participação na pesquisa, além de dois questionários (um para os coordenadores e, outro, para os docentes), elaborados pelos pesquisadores envolvidos com este estudo, para preenchimento on-line. Neles, solicitaram-se, aos coordenadores, o Projeto Político Pedagógico (PPP) do curso, bem como a ementa da(s) disciplina(s) de História da Psicologia. Entretanto, dois coordenadores encaminharam apenas o PPP e, três, encaminharam apenas o ementário da disciplina. Não obteve-se resposta dos outros cinco coordenadores, a despeito de reenvio de e-mail mais outras vezes. Por consequência, entendeu-se que eles se recusaram a participar da pesquisa. Ao final, obteve-se uma amostra de cinco coordenadores. Em relação aos professores contatados, nenhum respondeu ou preencheu o questionário específico à sua prática docente de ensino da História da Psicologia. Compreende-se que essa recusa implicou em uma restrição à pesquisa. Destarte, os únicos resultados considerados foram os relativos aos cinco coordenadores que responderam aos questionários. Este fato, assim, reduziu as potencialidades explicativas dos resultados, mas não impossibilitou o cumprimento do objetivo proposto em relação às inferências que foram possíveis de ser levantadas.

A despeito disto, foi possível coletar algumas informações referentes aos dez cursos levantados, uma vez que todas as IES possuíam dados disponibilizados publicamente em seus sites, tais como matriz curricular, ementas e quadro docente. Este material foi de utilidade, pois não dependeu das respostas dos sujeitos convidados e nos possibilitou outra fonte de conhecimento.

Desta feita, o material coletado foi organizado de forma a proporcionar uma apreciação sobre a organização administrativa das IES, assim como sobre a forma como a História da Psicologia é organizada, nas IES pesquisadas.

\section{Resultados}

\section{Características dos cursos de graduação em Psicologia do estado do Mato Grosso do Sul}

Com base nos dados compilados em seguida, na Figura 1, observam-se algumas características gerais dos cursos de graduação em Psicologia, em MS, úteis ao entendimento das condições locais do ensino de História da Psicologia. 


\begin{tabular}{|c|c|c|c|c|}
\hline \multirow{2}{*}{ Cidade } & Sigla & $\begin{array}{c}\text { Natureza } \\
\text { Administrativa }\end{array}$ & $\begin{array}{c}\text { Organização } \\
\text { Administrativa }\end{array}$ & Fundação do Curso \\
\hline \multirow{2}{*}{ Campo Grande } & UCDB & Privada & Universidade & 1975 \\
\cline { 2 - 5 } & UNIDERP & Privada & Universidade & 2000 \\
\cline { 2 - 5 } & UNIGRAN & Privada & Centro Universitário & 2008 \\
\cline { 2 - 5 } & UFMS & Pública & Universidade & 2001 \\
\hline Corumbá & UFMS - CPAN & Pública & Universidade & 1967 \\
\hline Dourados & FAD & Privada & Universidade & 2006 \\
\cline { 2 - 5 } & UFGD & Pública & Universidade & 2009 \\
\cline { 2 - 5 } & UNIGRAN & Privada & Centro Universitário & 1998 \\
\hline Paranaíba & UFMS - CPAR & Pública & Universidade & 2001 \\
\hline Três L*agoas & AEMS & Privada & Faculdade & 2008 \\
\hline
\end{tabular}

Fonte: adaptação do estudo de Souza (2011).

Figura 1. Características gerais dos Cursos de Graduação em Psicologia, em MS.

Nesta Figura, visualizam-se que seis $(60 \%)$ cursos de graduação, em Psicologia, estão alocados em IES particulares do estado de MS. Esse resultado é coincidente aos estudos de Mancebo et al. (2015), os quais indicam que as expansões dos cursos de Psicologia ocorrem por meio da iniciativa privada, sobretudo nos interiores dos estados brasileiros. Nota-se, também, que oito IES (80\%) foram fundadas entre o final dos anos de 1990 e a década de 2000, coincidindo com políticas de expansão do Ensino Superior no Brasil (Castelo Branco \& Feitosa, 2017). Além disso, observa-se que seis IES (60\%) estão situadas em cidades no interior do estado; destas, cinco foram fundadas no já mencionado período de expansão das IES particulares. Em seguida, na Figura 2, organizam-se alguns dados, especificamente relacionados às cincos IES dos coordenadores que responderam ao questionário da pesquisa.

\begin{tabular}{|c|c|c|c|}
\hline Instituição & Regime & $\begin{array}{c}\text { Frequência de } \\
\text { Ingresso por ano }\end{array}$ & Período do dia \\
\hline I1 & Semestral & 02 & Matutino e Noturno \\
\hline I2 & Semestral & 01 & Matutino \\
\hline I3 & Semestral & 02 & Matutino e Noturno \\
\hline I4 & Semestral & 01 & Vespertino e Noturno \\
\hline I5 & Anual & 01 & Matutino e Vespertino \\
\hline
\end{tabular}

Figura 2. Características de organização dos cursos de graduação respondentes

Percebe-se, conforme a Figura 2, que quatro $(80 \%)$ das IES respondentes adotam um regime semestral. As I4 e I5, por serem instituições públicas, têm como ingresso o Sistema de Seleção Unificada (SISU). Nota-se, ainda, que a quantidade de turmas ingressantes varia entre uma e duas, por ano, sendo que o mais comum é o ingresso de apenas uma turma (60\%). Ainda segundo a Figura 2, entende-se que o curso de graduação, em Psicologia, é encontrado em todos os períodos do dia, mas essa disponibilidade é alterada de acordo com a IES. Os cursos são oferecidos, predominantemente, pela manhã (43\%), sendo que $28 \%$ deles ocorre apenas à noite. Este perfil de distribuição sugere o atendimento a certas camadas sociais que podem não ter um emprego formal - visto estarem em um curso integral - ou que podem ter empregos de meio horário - aqueles que estudam somente pela manhã. Esta característica parece marcar, historicamente, a constituição dos cursos de graduação, em Psicologia, no país, evidenciando uma relação com o fortalecimento 
das classes médias urbanas (Mancebo et al., 2015). Este segmento, portanto, constitui o grupo principal de alunos, do curso. Vale ressaltar que três das quatro $(66 \%)$ das instituições que ofertam o curso, no período matutino, também o oferecem no período noturno.
No que concerne à formação ofertada pelos cursos de graduação, segundo a Figura 3, exposta a seguir, expressa-se a configuração estabelecida pela Lei no. 4119, com a presença das três modalidades de formação: Bacharel, Psicólogo e Licenciado (Casa Civil, 1962).

\begin{tabular}{|c|c|c|c|}
\hline \multirow{2}{*}{ Instituição } & \multicolumn{3}{|c|}{ Modalidade de Formação } \\
\hline & Bacharelado & Psicologia & Licenciatura \\
\hline I1 & $\cdot$ & $\cdot$ & $\cdot$ \\
\hline $\mathrm{I} 2$ & $\cdot$ & $\cdot$ & $\cdot$ \\
\hline $\mathrm{I} 3$ & $\cdot$ & - & - \\
\hline $\mathrm{I} 4$ & - & - & \\
\hline I5 & $\cdot$ & - & \\
\hline
\end{tabular}

Figura 3. Modalidade de Formação das IES respondentes

Atenta-se para o fato de que apenas as IES particulares I1, I2 e I3 oferecem a formação em Licenciatura, perfazendo $60 \%$ do total dos estabelecimentos de ensino minutados. Possivelmente, isso ocorre em razão de um maior controle da gestão, com vistas às alterações realizadas pelas novas DCNs, para os cursos de graduação em Psicologia que passaram a exigir a oferta da Licenciatura (Ministério da Educação e Cultura, 2011). Inclusive, tal processo de implementação tem sido fonte de debates, pelo Brasil (Sekkel \& Barros, 2013).

\section{Sobre o ensino da disciplina de História da Psicologia nas instituições analisadas}

Adentrando as informações colhidas pelos questionários respondidos, na Figura 4, exposta a seguir, organizam-se algumas informações relacionadas às disciplinas de História da Psicologia.

\begin{tabular}{|c|c|l|}
\hline Instituição & $\begin{array}{c}\text { Número de } \\
\text { Disciplinas } \\
\text { Idenfiticadas }\end{array}$ & Nome(s) \\
\hline I1 & 02 & História da Psicologia I; História da Psicologia II. \\
\hline I2 & 01 & História da Psicologia. \\
\hline I3 & 02 & História da Psicologia; Ciência \& Profissão. \\
\hline I4 & 02 & Introdução à Psicologia I; Introdução à Psicologia II. \\
\hline I5 & 05 & $\begin{array}{l}\text { Introdução à Psicologia; Fundamentos Epistemológicos e Históricos da Psicologia: } \\
\text { Enfoque Comportamental; Fundamentos Epistemológicos e Históricos da Psicologia: } \\
\text { Enfoque Psicodinâmico; Fundamentos Epistemológicos e Históricos da Psicologia: } \\
\text { Enfoque Sócio-Histórico. }\end{array}$ \\
\hline
\end{tabular}

Figura 4. Caracterização das Disciplinas Identificadas como História da Psicologia pelas IES respondentes 
Observa-se que todas as cinco IES respondentes possuem disciplinas intituladas História da Psicologia ou com outra rubrica, como, por exemplo, Introdução à Psicologia. Isso sugere um atendimento à obrigatoriedade do ensino de fundamentos históricos e epistemológicos da Psicologia, conforme as DCNs de 2011 (Ministério da Educação e Cultura, 2011). Atenta-se, ainda, que quatro (80\%) IES possuem mais de uma disciplina em sua matriz curricular. Mesmo considerando os limites, ao se tecer generalizações para o restante de MS, ou mesmo do Brasil, a presença desse componente curricular, em todas as IES respondentes, possibilita indícios de um cenário diferente daquele indicado no Canadá e nos EUA (Barnes \& Greer, 2014). Melhor esclarecendo, enquanto nestes países o ensino de História da Psicologia não é obrigatório, no Brasil não apenas é imperativo, como tal prerrogativa parece estar sendo implementada. Assim, mesmo com uma amostra institucional reduzida, percebe-se a presença clara do ensino de História da Psicologia, com eventuais ocorrências de mais de uma disciplina, em algumas matrizes curriculares.

Ainda na Figura 4, considerando os nomes das disciplinas, aponta-se certa similaridade entre as IES investigadas, sendo que os nomes História da Psicologia e Introdução à Psicologia são os mais comuns, nas matrizes. O termo "Introdução", ademais, pode ter relação com o momento em que as disciplinas são inseridas, no curso. Para os cursos de graduação, com regime de ingresso semestral, as disciplinas identificadas com "I" e "II" ocorrem, respectivamente, nos dois primeiros semestres; para aqueles com regime anual, o ensino de tais disciplinas acontece no primeiro ano. Assim, a disciplina seria introdutória às temáticas e teorias psicológicas, recepcionando os estudantes no início de sua formação.

No caso da I5, seu coordenador sugere que a História da Psicologia funciona como uma ferramenta de ensino para diferentes abordagens teóricas da Psicologia. Além de uma introdução geral ao campo da diversidade da Psicologia, os fundamentos teóricos e históricos seriam compartimentados, contribuindo para a compreensão de algumas abordagens psicológicas. Para o coordenador da I2, a disciplina permite ao estudante aprender como a História e a Filosofia contribuem para a compreensão da evolução da Psicologia moderna, como ciência. O coordenador da I4, nessa mesma direção, menciona que, baseado no conteúdo da matéria, "a disciplina Introdução à Psicologia I deveria ter por nome História da Psicologia”, indicando que a disciplina é fundamental, porque mostra o desenvolvimento da Psicologia.

Além do quesito introdutório, os textos dos PPPs e os ementários dos cursos de Psicologia sugerem que é função da História da Psicologia o desenvolvimento do pensamento crítico. A promoção de tal pensamento varia de acordo com a instituição, com influência de diferentes matrizes ou áreas da Psicologia. As instituições I3 e I4, por exemplo, atentaram para áreas específicas, como a Psicologia da Saúde. Assim, a História da Psicologia poderia cumprir o papel de levar os estudantes a criarem conexões entre passado e presente, a fim de compreender a relação entre Psicologia e Saúde, ao longo do tempo. Isso se desdobraria, inclusive, em um maior tempo despendido com disciplinas que abordassem os aspectos históricos da Psicologia. A título de exemplo, cite-se a I5, que apresenta disciplinas vinculadas a diferentes correntes psicológicas. As outras instituições (I1 e I2), por sua vez, apresentam uma preocupação em despertar discussões entre os alunos, contribuindo para a formação de um senso crítico. Esse comportamento é promovido de maneiras diferentes, em cada instituição. Em I1, há o incentivo à pesquisa e um discurso de aliar a teoria à prática; em I2, considera-se que o foco deve ser na aprendizagem, em que o professor age como mediador e o aluno é ativo, no processo de ensino. De qualquer modo, os propósitos do ensino visam à formação de profissionais capacitados para aliar diversas áreas do conhecimento e, a partir disto, questionar certas realidades.

Dentro das disciplinas computadas, ainda é possível encontrar uma atenção para a formação do campo científico da Psicologia e a exposição de algumas perspectivas de pensamento, difundidas até hoje. Tal fato se acha presente nos documentos que foram fornecidos pelas coordenações de I1, I2, I4 e I5, que apresentam os conteúdos a serem ministrados para cada disciplina, além de expor, a saber: a bibliografia básica e complementar, utilizada nessas disciplinas; os conteúdos programáticos essenciais como, por exemplo, o modo pelo qual a Psicologia se constituiu e seus reflexos atuais. As ementas, de maneira geral, buscam refletir a forma em que a Psicologia se constituiu como Ciência, em suas diversas ramificações.

Sobre a bibliografia encontrada nos dados fornecidos pelos coordenadores anuentes, observamos um total de 65 obras, pertencentes aos cursos em I1, I2, I4 e I5. Ressalta-se que I3 não teve seu material 
contabilizado, pela ausência dos documentos relativos à disciplina. Em razão da forte presença de disciplinas relacionadas ao campo da História da Psicologia, na I5, a maioria das obras contabilizadas era exclusiva desta instituição, perfazendo 56.

Dos 65 materiais bibliográficos investigados, apenas dois foram utilizados em todas as instituições analisadas, ou seja: Psicologias: Uma introdução ao estudo da Psicologia (1999), de Ana Bock, Odair Furtado e Maria Teixeira, e História da Psicologia Moderna (1992), de Duane Schultz e Sydney Schultz. Além disso, uma obra foi utilizada por três instituições, Matrizes do Pensamento Psicológico (1991), de Luís Cláudio Figueiredo. Organizam-se na Figura 5, expressa em seguida, as seis referências bibliográficas mencionadas em duas instituições simultaneamente. Desses seis livros, cinco são produções nacionais, dos quais quatro são claramente vinculados a historiadores da Psicologia atuantes no Brasil, como Ana Maria Jacó-Vilela e Marina Massimi. Concernente ao ano de publicação do material pesquisado, nota-se que existem referências datadas a partir da década de 1960 até 2015, havendo uma grande concentração de obras editadas nos anos 2000.

\begin{tabular}{|c|c|c|}
\hline Autor(es) & Título & Ano de Publicação \\
\hline $\begin{array}{c}\text { Bock, A., Gonçalves, M., \& } \\
\text { Furtado, O. }\end{array}$ & $\begin{array}{c}\text { Psicologia sócio-histórica: } \\
\text { uma perspectiva crítica da Psicologia }\end{array}$ & 2001 \\
\hline Figueiredo, L, \& Santi, P. & Psicologia: uma (nova) introdução & 2004 \\
\hline Freire, I. & Raízes da Psicologia & 1997 \\
\hline $\begin{array}{c}\text { Jacó-Vilela, A., Ferreira, A., \& } \\
\text { Portugal, F. }\end{array}$ & $\begin{array}{c}\text { História da Psicologia: } \\
\text { rumos e percursos }\end{array}$ & 2005 \\
\hline Luria, A. & $\begin{array}{c}\text { Curso de Psicologia geral: } \\
\text { introdução evolucionista à Psicologia }\end{array}$ & 1979 \\
\hline Massimi, M. & História da Psicologia brasileira: da época colonial até 1934 & 1990 \\
\hline
\end{tabular}

Figura 5. Referências encontradas, simultaneamente, em duas IES

Do transcurso dos resultados obtidos, pondera-se que a disciplina de História da Psicologia exerce um papel formativo importante para o esclarecimento da identidade e da diferença das correntes psicológicas e se configura como um recurso introdutório às questões conceituais e metodológicas da Psicologia, desde o seu surgimento, até suas implicações nos campos profissionais. A História da Psicologia, destarte, representa uma importante ferramenta para melhor compreender a Psicologia, assim como para contribuir com críticas ao presente (Fierro, 2014; Krampen, 2015; Merced, Stutman \& Mann, 2017; Vaugh-Blount et al., 2009). Em um olhar para o passado, é possível identificar diferentes características dos campos teórico-metodológicos da Psicologia, aquilo que se mantém e poderia permanecer, assim como aquilo que deveria ser modificado. Neste cenário, a História da Psicologia vem sendo compreendida como uma disciplina capaz de auxiliar na formação crítica de psicólogos. Todavia, isso nem sempre ocorre de maneira tranquila, uma vez que ela pode ser caracterizada como um estudo desinteressante para os alunos, sob a acusação de não apresentar uma utilidade prática, além de se encontrar defasada das psicologias e questões profissionais mais atuais, o que pode, eventualmente, levar a um descaso por parte da administração dos cursos (Stevison et al., 2010).

No ensino da História da Psicologia, baseado no pensamento de Gomes (1996), aponta-se as dificuldades de se promover, em uma única disciplina, o que é introdutório e basilar, mesclado com aquilo que é intermediário, diferenciador e integrador e aquilo que é avançado e profissionalizante. Outra dificuldade seria a de condensar vários conteúdos históricos distintos, em uma única disciplina, dado que são poucas as instituições que oferecem mais de uma.

Com efeito, não milita-se a favor da inclusão de mais disciplinas históricas da Psicologia, em IES, mas argumenta-se que a restrição de sua oferta - ou a falta de sua articulação com outras disciplinas - pode gerar omissão e controvérsia sobre as fundações, fundamentações e aplicações da Psicologia. Segundo ajuíza-se, o ensino da História da Psicologia deve considerar essas 
questões e articulações, com vistas a proporcionar uma iniciação que enfatize as identidades e as diferenças, bem como as relações e os contrastes dos conhecimentos psicológicos. Logo, indica-se que é possível sincronizar os conteúdos relacionados à História da Psicologia com outras disciplinas, como a Psicologia Social, a Psicologia Experimental, a Avaliação Psicológica, a Psicologia do Desenvolvimento, etc. Esse planejamento acarretaria em uma outra forma de organizar e difundir o ensino da História da Psicologia em outras disciplinas, contribuindo como conteúdo inicial para o seu desenvolvimento, porém respeitando o desenvolvimento ulterior, nas teorias e práticas específicas a cada disciplina.

Outra discussão que se indica, novamente inspirados por Gomes (1996), ocorre em relação à inclusão dos aspectos históricos da profissionalização da Psicologia, no Brasil, desde a sua regulamentação, em 1962, até suas articulações atuais com os campos da Saúde. Embora profícuos e importantes à iniciação formativa do psicólogo, ajuíza-se que outros pontos, oriundos da Filosofia da Ciência e frequentemente associados à História da Psicologia, contribuem com um pensamento organizador e crítico da Psicologia em sua pluralidade conceitual, metodológica e prática. Em referência aos pontos anteriormente citados, vale ressaltar que eles se referem às implicações da Ontologia, da Epistemologia, da Lógica e da Ética, em questões concernentes à História da Psicologia. No domínio ontológico, questiona-se: O que seria a Psicologia? Qual(is) o(s) seu(s) objeto(s) de estudo? Qual(is) a(s) sua(s) natureza(s)? Na esfera epistemológica: Em que consiste o objeto psicológico? Quais são os modos de acesso e apreensão do psiquismo e do conhecimento psicológico? Como verificá-los e avaliá-los como válidos? No campo lógico: Quais são as justificativas que argumentam os pressupostos e as conclusões de cada corrente psicológica? Como elas se relacionam, a partir de tensões e complementações? No quesito ético: Como as aplicações e expressões dos conhecimentos psicológicos afetam a prática do psicólogo?

Conquanto essas questões sejam extensas e difíceis de ser condensadas e sustentadas, didaticamente, em poucas disciplinas, seja para um público iniciante ou seja como pauta introdutória de outros componentes curriculares, reconhecemos que o ensino da História da Psicologia é um campo aberto à criatividade de quem ministra esse conhecimento. Os pontos anteriormente mencionados podem sugerir um horizonte relacional, entre professor e aluno, que desperte o interesse por esta disciplina. Ressalta-se que, para além de uma disciplina, a História da Psicologia, ainda, é um campo de pesquisas que congrega vários pesquisadores, que produzem conhecimento e (re)pensam a Psicologia em suas diversas manifestações científicas, profissionais e propagações locais. Portanto, a disciplina de História da Psicologia possibilita o acesso a esse campo, circunscrito a outras institucionalizações dispostas em sociedades, grupos de trabalho, eventos e produções acadêmicas (artigos, livros, dissertações e teses).

\section{Discussão}

O estudo da História da Psicologia, como uma disciplina ofertada em cursos de Psicologia, representa uma perspectiva para o melhor entendimento de como esse saber se organiza, institucionalmente, no Brasil. Nesta perspectiva, esta disciplina, além de compor uma introdução às bases da Psicologia moderna, auxilia na formação crítica de psicólogos e preconiza, potencialmente, um interesse formativo pela historiografia da Psicologia. Ao perceber a relevância disso, pesquisou-se essa realidade em um contexto mais próximo, de modo a entender a situação da História da Psicologia, como disciplina, em cursos de graduação em Psicologia, em IES, em MS.

Devido ao número de respostas obtidas, a pesquisa ficou restrita ao contexto institucional expresso pelos coordenadores de cinco IES, não sendo possível analisar a percepção dos professores responsáveis pela disciplina. Outra limitação concerniu ao fato de que os dados fornecidos pelos coordenadores anuentes somente dizem respeito às disciplinas de nome História da Psicologia ou Introdução à Psicologia, não tateando outros conteúdos históricos que poderiam estar disponíveis, em outras disciplinas. Apesar disso, elencaram-se alguns aspectos que julgamos relevantes ao entendimento da organização institucional do ensino da História da Psicologia, no estado.

Primeiramente, todas as instituições convidadas e respondentes da pesquisa possuíam disciplinas relacionadas à História da Psicologia, o que possibilitou indícios de que os cursos seguem elementos preconizados pelas DCNs, no que reporta ao ensino da História da Psicologia, na formação do psicólogo. Esses elementos apareceram nos textos dos PPPs, a nós enviados e, também, naquilo que os coordenadores preencheram. Em segundo lugar, nota-se que as 
disciplinas de História se encontravam no início dos cursos. Isso sugere que se considerava ser o seu estudo uma possibilidade de apresentação geral das diferentes perspectivas da Psicologia. Essa característica pode indicar, também, que tais disciplinas constituíam pré-requisitos para aquelas específicas de teorias, métodos e técnicas psicológicas. Em terceiro lugar, percebe-se a presença de livros-textos diretamente relacionados à História da Psicologia, com a assinatura de vários autores brasileiros. Isso demonstra uma preocupação de utilizar referências nacionais de autores que se debruçaram sobre as questões históricas da Psicologia.

Por fim, para uma visão mais ampla da organização nacional da História da Psicologia, como disciplina e recurso formativo de psicólogos, estima-se que novos estudos sejam realizados, em outras localidades brasileiras e em cursos de pós-graduação, com a variação dos instrumentos utilizados como, por exemplo, a análise documental de PPPs e dos planos de ensino.

\section{Referências}

Barnes, M., \& Greer, S. (2014). Does the future have a history of psychology? A report on teaching, research, and faculty positions in Canadian universities. History of Psychology, 17(2), 159-169.

Casa Civil (1962). Lei No. 4.119 - Dispõe sobre os cursos de formaşão em Psicologia e regulamenta a profissão de psicólogo. Brasília, DC. Recuperado de: http://www.planalto.gov. br/ccivil_03/leis/1950-1969/L4119.htm.

Castelo Branco, P., \& Feitosa, E. (2017). Formação do psicólogo nos interiores do Brasil: reflexões e implicações. In: Lemos, F. (Org.), Conversas tranversalizantes entre psicologia política, social-comunitária e institucional com os campos da educaşão, saúde e direitos, v.7 (pp.557-565). Curitiba: Editora CRV.

Fierro, C. (2014). La historia de la psicología como curso de grado: Sentido formativo y recursos para su enseñanza. Revista Puertorriqueña de Psicologia, 25(2), 396-409.

Gomes, W. (1996). História da Psicologia - Pesquisa Formação - Ensino. In: História da Psicologia para curso de Graduação (pp. 150-159). São Paulo: EDPUC.
Gundlach, H. (2012). A Psicologia como Ciência e como Disciplina: o caso da Alemanha. In: S. Araújo (Org.), História e filosofia da psicologia: perspectivas contemporâneas (pp. 133-165, S. Araújo, Trad.). Juiz de Fora, MG: UFJF.

Krampen, G. (2015). Scientometric trend analysis of publications on the history of psychology: Is psychology becoming an unhistorical Science? Scientometrics, 106(3), 1217-1238.

Loureiro, I., \& Baptista, M. (2007). A História da Psicologia como disciplina de mestrado: relato de uma experiência. Memorandum, 12, 143-151.

Mancebo, D., Vale, A., \& Martins, T. (2015). Políticas de expansão da educação superior no Brasil, 1995-2010. Revista Brasileira de Educaşão, 20(60), 31-50.

Naves, F., Silva, S., Peretta, A., Nasciutti, F., \& Silva, L. (2017). Formação de psicólogos para a educação: concepções de docentes. Psicologia da Educação, (44), 67-77. $10.5935 / 2175-3520.20170007$

Merced, M., Stutman, Z., \& Mann, S. (2017). Teaching the history of psychology: A content analysis of course syllabi from Doctor of Psychology Programs. Psychology: Learning and Teaching, 1(1), 01-16. 10.1177/1475725717729909

Ministério da Educação e Cultura (2011). Institui as Diretrizes Curriculares Nacionais para os cursos de graduação em Psicologia. Brasília, DC. Recuperado de: http://portal.mec.gov.br/index.php?option $=$ com docman\&view $=$ download\&alias $=7692-$ rces005-11pdf\&category_slug $=$ marco-2011-pdf\&Itemid $=30192$.

Sekkel, M., \& Barros, C. (2013) (Orgs.). Licenciatura em Psicologia: Temas atuais. São Paulo: Zagadoni Editora.

Stevison, B., Biggs, P., \& Abramson, C. (2010). Google Earth as a source of ancillary material in a History of Psychology class. Psychological Reports, 106(3), 665-670.

Souza, F. (2011). Por uma história da Análise do Comportamento no Mato Grosso do Sul. 69f. Dissertação de mestrado, Programa de Estudos Pós-graduados em Psicologia Experimental: Análise do Comportamento, Pontifícia Universidade Católica de São Paulo. São Paulo, SP.

Vaughn-Blount, K., Rutherford, A., Baker, D., \& Johnson, D. (2009). History's mysteries, demystified: Becoming a psychologist-historian. The American Journal of Psychology, 122(1), 117-129.

Recebido em: 21 de novembro de 2017 Aprovado em: 05 de junho de 2020 